\title{
Mixing Enthalpy Measurements of Liquid Fe-Ti Alloys by Levita- tion Alloying Calorimetry and Calculation of the Thermodynamic Properties of Mixing
}

\author{
Uwe THIEDEMANN, Jianping OIN, Klaus SCHAEFERS, Michael RÖSNER-KUHN \\ and Martin G. FROHBERG
}

Institute of Metallurgy, Technical University Berlin, Joachimstaler Str. 31/32, D-10719 Berlin, Germany.

(Received on March 9, 1995; accepted in final form on July 24, 1995)

\begin{abstract}
Mixing enthalpies, $\Delta H^{m}$, of liquid iron-titanium alloys were measured in dependence of the composition by levitation alloying calorimetry. The measurements were carried out at $T=1950 \mathrm{~K}$ up to $x_{\mathrm{Ti}}=0.42$ in the iron rich concentration range and at $T=2112 \mathrm{~K}$ up to $x_{\mathrm{Fe}}=0.31$ in the titanium rich concentration range. The mixing enthalpy was found to be exothermic. The temperature dependence of $\Delta H^{m}$ could be described with help of the regular associate model. On the basis of an adapted function for the mixing enthalpy calculations of the excess heat capacity, the free energy of mixing, the partial free energies of mixing and the activities of iron and titanium are performed. The comparison of the calculated activities with corresponding experimental data from the literature shows a good accordance.
\end{abstract}

KEY WORDS: liquid iron-titanium alloys; levitation alloying calorimetry; mixing enthalpy measurements; regular associate model; excess heat capacity; free energy of mixing; partial free energy of mixing; activities.

\section{Introduction}

In steelmaking processes the system $\mathrm{Fe}-\mathrm{Ti}$ is important in connection with the ferrotitanium-hardening alloys and the titanium alloyed steels. The intermetallic compound FeTi can be used as a hydrogen storing material. ${ }^{1)}$ Moreover, the alloy $\mathrm{Fe}_{84} \mathrm{Ti}_{16}$ can be transformed into metallic glass by melt quenching. ${ }^{2)}$ The phase diagram of the system exhibits two intermetallic phases $^{3)}$ : the compound FeTi ( $\mathrm{CsCl}$ structure) incongruent melting with a peritectic temperature of $T=$ $1590 \mathrm{~K}$, and the congruent melting Laves phase $\mathrm{Fe}_{2} \mathrm{Ti}$ with a liquidus temperature of $T=1700 \mathrm{~K}$.

In consequence of atomic interactions frequently chemical short range order occurs in the liquid state of alloys. The compositions of the short range ordered volume elements in the melt mostly correspond with the stoichiometry of a solid stable compound which can be found in the phase diagram. ${ }^{4)}$

According to Sommer ${ }^{5}$ the occurrence of short range order is a necessary prerequisite for the glass formation ability of alloys by rapid solidification from the liquid state. The amount of the mixing enthalpy, $\Delta H^{m}$, represents the strength of the compound forming tendency. For alloys which tend to form short range ordered volume elements with favoured compositions, the course of the mixing enthalpy can furnish information about the stoichiometry of these volume elements.

The levitation alloying calorimetry (LAC) makes it possible to levitate and alloy metallic samples in the liquid state. The containerless working LAC-method is suitable for experiments at high temperatures ${ }^{6-11)}$ and strong reactive metals. ${ }^{12)}$ For the system presented here the mixing enthalpy in the titanium rich range could be measured for the first time. The mathematical adaptation of the experimental data by thermodynamic models makes it possible to calculate other thermodynamic properties like the free energy of mixing, the partial free energies of mixing and the activities.

\section{Experimental Equipment}

The LAC-equipment is shown schematically in Fig. 1.

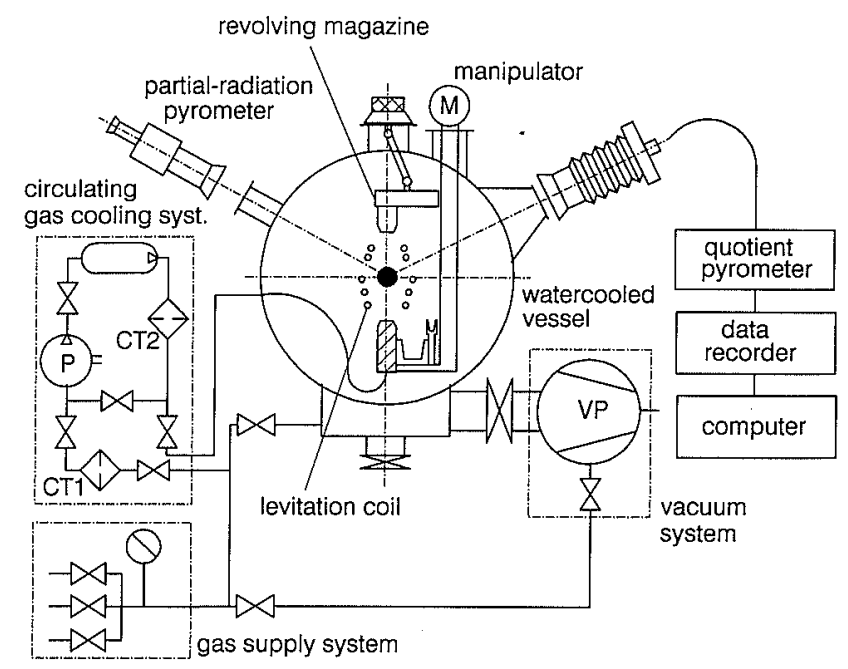

Fig. 1. Schematic LAC equipment. 
It consists of four major parts.

- The closed water cooled vessel includes an electromagnetic coil to levitate and to melt the basis sample, a revolving magazine to hold 16 solid secondary samples and a manipulator to position the basis sample in the middle of the coil before levitation starts and to move the nozzle of the circulating gas cooling system just below the sample.

- The temperature recording system consists of a quotient pyrometer, a data recorder, a translator and a computer.

- The vacuum and the gas supply system (helium) are installed to avoid oxidation of the sample.

- The circulating gas cooling system guarantees a precise temperature control of the sample during the experiment.

The experiment is carried out by levitating and melting a basis sample $A$ in the electromagnetic field of a levitation coil. With help of the circulating gas cooling system (variable gas flow) the temperature of the sample is adjusted to the initial experimental temperature after each alloying step. By turning the revolving magazine secondary samples B are gradually alloyed to the basis sample. During each alloying process the resulting temperature change is continuously measured by the quotient pyrometer and registered by the data recorder.

\section{Evaluation and Problem}

The temperature-time curve of an alloying process is shown schematically in Fig. 2. It is necessary to ascertain the real thermodynamic temperature difference $\Delta T_{L}$ to evaluate the curve on the basis of an energy balance. The idea is to assume an instantaneous fall of the sample temperature to the lowest value $T^{*}$ at a constant time $t^{*}$. For this the segment $\mathrm{G}-\mathrm{W}$ of the temperature-time curve is extended to the point $\mathrm{D}$ and $\Delta T_{L}$ is determined by a mathematical treatment where the hatched areas have to be equal. This mathematical treatment has been developed in the following way ${ }^{11,13)}$ : merging two samples of the same metal no mixing enthalpy occurs $\left(\Delta h^{m}=0\right)$ so that a theoretical temperature difference $\Delta T_{L, \text { theor. }}$ can be calculated from the general energy balance of the alloying process:

$$
\Delta h_{L}+\Delta h_{B}+\Delta h^{m}=0
$$

$\Delta h_{L}$ is the total mixing effect. $\Delta h_{B}$ is the enthalpy change

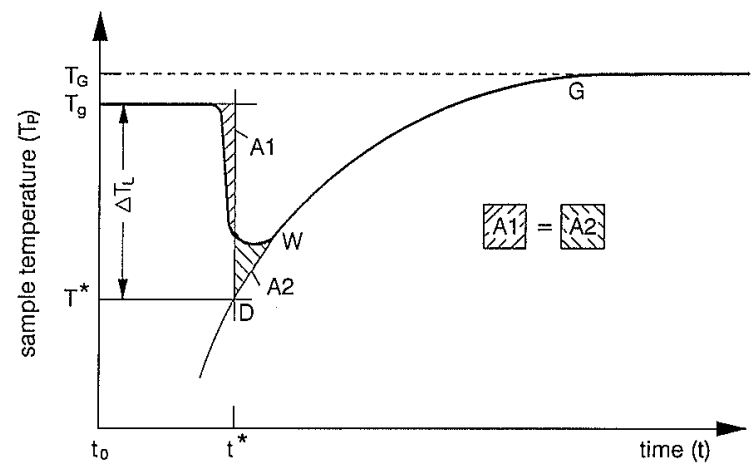

Fig. 2. Schematic time-temperature curve of an alloying process. of the secondary sample caused by its temperature rise from $T_{R}=298 \mathrm{~K}$ (room temperature) to the experimental temperature $T_{g}$ during the alloying process. For the special case $\Delta h^{m}=0 \Delta T_{L, \text { theor, }}$ should be equal to the experimental value $\Delta T_{L}$. Such measurements have been carried out with $\mathrm{V}-\mathrm{V}$ and $\mathrm{Ni}-\mathrm{Ni} .^{11)}$ This procedure delivers a basic function (Eq. (2)) for the extrapolation of the temperature rise $\mathrm{G}-\mathrm{W}$ (Fig. 2), so that a reliable evaluation of $\Delta T_{L}$ is possible in the mathematical form:

$$
T=a_{0}+a_{1} t^{0.2}+a_{2} t^{1.2}
$$

The single terms of Eq. (1) in detail yield:

$$
\begin{aligned}
& \Delta h_{L}=\left(n_{A}+n_{B}\right) C_{p}^{A B} \Delta T_{L} \\
& \Delta h_{B}=n_{B}\left[H_{B}\left(T_{g}\right)-H_{B}\left(T_{R}\right)\right] \\
& \Delta h^{m}=-\left\{n_{B}\left[H_{B}\left(T_{g}\right)-H_{B}\left(T_{R}\right)\right]+\left(n_{A}+n_{B}\right) C_{p}^{A B} \Delta T_{L}\right\}
\end{aligned}
$$

$n_{A}$ and $n_{B}$ are the mole numbers, $H_{B}\left(T_{g}\right)$ and $H_{B}\left(T_{R}\right)$ are the enthalpies of the secondary sample at the experimental temperature and at room temperature respectively and $C_{p}^{A B}$ is the heat capacity of the alloy.

The enthalpy $\Delta h_{j}^{m}$ and the molar enthalpy $\Delta H_{j}^{m}$ after $j$-additions of secondary samples can be calculated as follows:

$$
\begin{aligned}
\Delta h_{j}^{m}=- & \sum_{i=1}^{i=j}\left\{n_{B i}\left[H_{B}\left(T_{g}\right)-H_{B}\left(T_{R}\right)\right]\right. \\
& \left.+\left(n_{A}+\sum_{m=1}^{m=i} n_{B m}\right) C_{p i}^{A B} \Delta T_{L i}\right\} \\
\Delta H_{j}^{m}= & \frac{\Delta h_{j}^{m}}{n_{A}+\sum_{i=1}^{i=j} n_{B i}} \ldots \ldots \ldots \ldots \ldots \ldots \ldots \ldots \ldots \ldots \ldots \ldots \ldots
\end{aligned}
$$

All the values in the Eqs. (6) and (7) except $C_{p i}^{A B}$ can be obtained from the experiments and the literature. The heat capacity of an alloy $\mathrm{AB}$ is given as follows:

$$
C_{p}^{A B}=C_{p}^{A B}(i d)+\Delta C_{p}^{A B}(x s)
$$

with Neumann-Kopp's rule for the ideal heat capacity

$$
C_{p}^{A B}(i d)=x_{A} C_{p}^{A}+x_{B} C_{p}^{B}
$$

$C_{p}^{A}$ and $C_{p}^{B}$ are the heat capacities of the pure metals $\mathrm{A}$ and $\mathrm{B}, \Delta C_{p}^{A B}(x s)$ is the excess heat capacity of the alloy. Combination of the Eqs. (6) to (8) yields:

$$
\begin{aligned}
\Delta H_{j}^{m}= & \left(n_{A}+\sum_{i=1}^{i=j} n_{B i}\right)^{-1}\left\{-\sum_{i=1}^{i=j}\left[n_{B i}\left[H_{B}\left(T_{g}\right)-H_{B}\left(T_{R}\right)\right]\right.\right. \\
& +\left(n_{A}+\sum_{m=1}^{m=i} n_{B m}\right)\left(C_{p_{i}}^{A B}(i d) \Delta T_{L i}\right) \\
& \left.\left.+\left(n_{A}+\sum_{m=1}^{m=i} n_{B m}\right)\left(\Delta C_{p_{i}}^{A B}(x s) \Delta T_{L i}\right)\right]\right\} \ldots \ldots . .(10)
\end{aligned}
$$

$\Delta C_{p}^{A B}(x s)$ and its changes with temperature and composition are normally unknown. The general definition of the excess heat capacity is:

$$
\Delta C_{p}^{A B}(x s)=\left(\frac{d\left(\Delta H^{m}\right)}{d T}\right)_{p}
$$




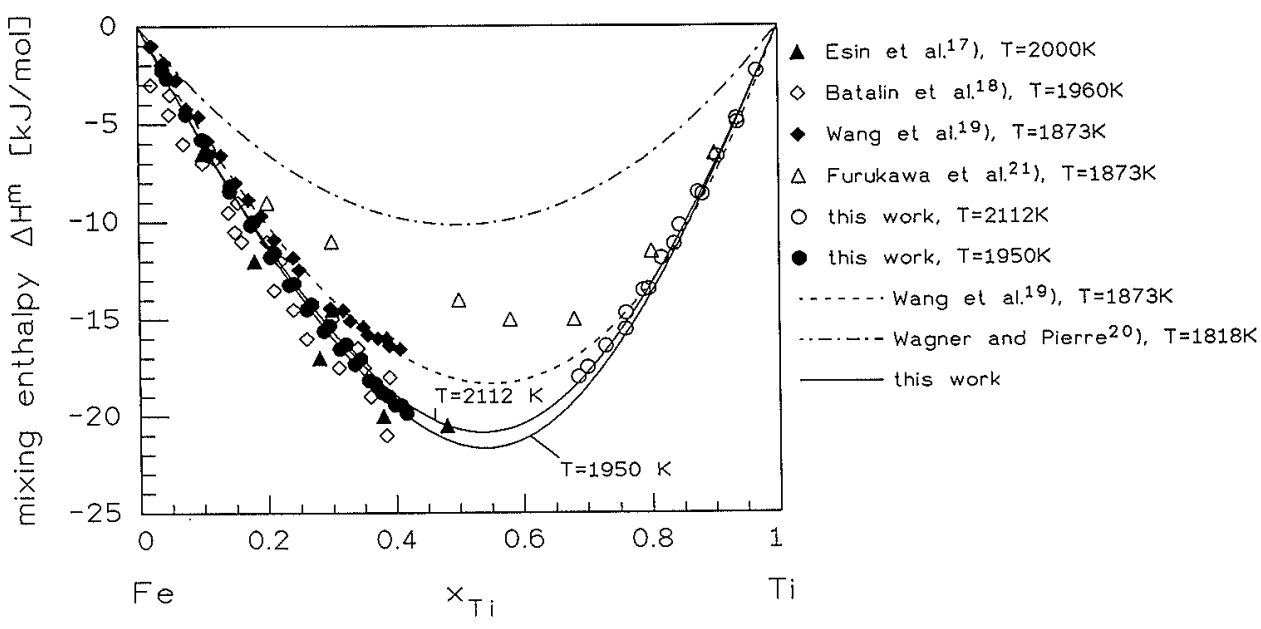

Fig. 3. Mixing enthalpies $\Delta H^{m}$ of liquid iron-titanium alloys.

A method to consider the excess heat capacity for the evaluation of the experimental data is given in. ${ }^{14)}$

The mixing enthalpies obtained by the procedure mentioned above were described with help of the regular associate model. According to the basic idea of this model the liquid binary alloy is regarded as a ternary solution of randomly distributed A and B atoms which are in equilibrium with associates having a definite stoichiometric composition $A_{i} B_{j}$. The associates can be understood as chemical short range ordered volume elements. The equilibrium constant of the associate formation is given as follows ${ }^{5)}$ :

$$
\begin{aligned}
& K=\frac{\gamma_{A_{i} B_{j}} x_{A_{i} B_{j}}}{\left(\gamma_{A_{f}} x_{A_{f}}\right)^{i}\left(\gamma_{B_{f}} x_{B_{f}}\right)^{j}}=\exp \left(-\frac{\Delta G_{A_{i} B_{j}}^{F}}{R T}\right) \\
& =\exp \left(\frac{\Delta H_{A_{i} B_{j}}^{F}-T \Delta S_{A_{i} B_{j}}^{F}}{R T}\right)
\end{aligned}
$$

$\gamma_{A_{i}}, \gamma_{B_{j}}$ and $\gamma_{A_{i} B_{j}}$ are the activity coefficients, $x_{A_{f}}, x_{B_{f}}$ and $x_{A_{i} B_{j}}$ are the mole fractions in the associated solution and $\Delta G_{A_{i} B_{j}}^{F}, \Delta H_{A_{i} B_{j}}^{F}$ and $\Delta S_{A_{i} B_{j}}^{F}$ are the molar free energy, the molar enthalpy and the molar entropy of formation of the associate.

Due to the minimum of $\Delta H^{m}(\sim 50 \mathrm{at} \%$ iron) (Fig. 3) we have assumed the associate FeTi. The activity coefficients $\gamma_{\mathrm{Fe}}, \gamma_{\mathrm{Ti}}$ and $\gamma_{\mathrm{FeTi}}$ were calculated according to Jordan's ${ }^{15)}$ Eqs. (13)-(15):

$$
\ln \gamma_{A_{i} B_{j}}=\frac{1}{R T}\left[x_{A_{f}}^{2} C_{2}+x_{B_{f}}^{2} C_{3}+x_{A_{f}} x_{B_{f}}\left(C_{2}+C_{3}-C_{1}\right)\right]
$$

$$
\ln \gamma_{A_{i}}=\frac{1}{R T}\left[x_{B_{f}}^{2} C_{1}+x_{A_{i} B_{j}}^{2} C_{2}+x_{B_{f}} x_{A_{i} B_{j}}\left(C_{1}+C_{2}-C_{3}\right)\right]
$$

$\ln \gamma_{B_{i}}=\frac{1}{R T}\left[x_{A_{f}}^{2} C_{1}+x_{A_{i} B_{j}}^{2} C_{2}+x_{A_{f}} x_{A_{i} B_{j}}\left(C_{1}+C_{3}-C_{2}\right)\right]$

The mixing enthalpy and entropy are given as follows:

$$
\begin{aligned}
& \Delta H^{m}=\frac{n_{A_{f}} n_{B_{f}}}{n} C_{1}+\frac{n_{A_{f}} n_{A_{i} B_{j}}}{n} C_{2}+\frac{n_{B_{f}} n_{A_{i} B_{j}}}{n} C_{3} \\
& +n_{A_{i} B_{j}} \Delta H_{A_{i} B_{j}}^{F} \\
& \Delta S^{m}=-R\left(n_{A_{f}} \cdot \ln x_{A_{f}}+n_{B_{f}} \cdot \ln x_{B_{f}}+n_{A_{i} B_{j}} \cdot \ln x_{A_{i} B_{j}}\right) \\
& +n_{A_{i} B_{j}} \Delta S_{A_{i} B_{j}}^{R}
\end{aligned}
$$

$n_{A_{f}}, n_{B_{f}}$ and $n_{A_{i} B_{j}}$ are the mole numbers of the free atoms $A_{f}, B_{f}$ and the associate $A_{i} B_{j} . n$ is the total mole number. The parameters $C_{1}, C_{2}$ and $C_{3}$ describe the interactions between $A_{f}-B_{f}, A_{f}-A_{i} B_{j}$ and $B_{f}-A_{i} B_{j}$.

With the help of an iteration method ${ }^{16)}$ we obtain the parameters $C_{1}, C_{2}, C_{3}, \Delta H_{A_{i} B_{j}}^{F}$ and $K$ by fitting the model parameters to the experimental values. Now $\Delta C_{p_{j}}^{A B}(x s)$ is calculated according to Eq. (11). $d\left[\Delta H_{j}^{m}\right] / d n_{A_{i} B_{j}}$ and $d n_{A_{i} B_{j}} / d T$ are derived ${ }^{16)}$ from Eqs. (12) and (16):

$$
\begin{aligned}
& \frac{d\left[\Delta H_{j}^{m}\right]}{d n_{A_{i} B_{j}}}=\left[-j x_{A_{f}}-i x_{B_{f}}-x_{A_{f}} x_{B_{f}}(1-i-j)\right] C_{1} \\
& +\left[x_{A_{f}}-i x_{A_{i} B_{j}}-x_{A_{f}} x_{A_{i} B_{f}}(1-i-j)\right] C_{2} \\
& +\left[x_{B_{f}}-j x_{A_{i} B_{j}}-x_{B_{f}} x_{A_{i} B_{f}}(1-i-j)\right] C_{3} \\
& +\Delta H_{A_{i} B_{j}}^{F} \\
& \frac{d n_{A_{i} B_{j}}}{d T}=\frac{n R}{2}\left(\frac{\Delta H_{A_{i} B_{j}}^{F}}{R T}+\ln \gamma_{A_{i} B_{j}}-i \cdot \ln \gamma_{A_{f}}-j \cdot \ln \gamma_{B_{f}}\right) \\
& \left\{\begin{array}{l}
{[i j+(1-i-j)} \\
\left.\cdot\left[i x_{a_{f}}+j x_{A_{f}}+(1-i-j) x_{A_{f}} x_{B_{f}}\right]\right] \cdot C_{1} \\
+[-i+(1-i-j) \\
\left.\quad \cdot\left[i x_{A_{i} B_{j}}-x_{A_{f}}+(1-i-j) x_{A_{f}} x_{A_{i} B_{j}}\right]\right] \cdot C_{2} \\
+[-j+(1-i-j) \\
\left.\cdot\left[j x_{A_{i} B_{j}}-x_{B_{f}}+(1-i-j) x_{B_{f}} x_{A_{i} B_{j}}\right]\right] \cdot C_{3} \\
+\frac{R T}{2}\left[\frac{1}{x_{A_{i} B_{j}}}+\frac{i^{2}}{x_{A_{f}}}+\frac{j^{2}}{x_{B_{f}}}-(1-i-j)^{2}\right]
\end{array}\right\}
\end{aligned}
$$

\section{Results and Discussion}

Figure 3 shows the results of our mixing enthalpy measurements and the literature data. The occurring error of the measurements is $\pm 10 \% . \Delta H^{m}$ of the system 
is exothermic. The measurements in the iron rich concentration range are in accordance with the mixing enthalpies given by Esin et al., ${ }^{17)}$ Batalin et al. ${ }^{18)}$ and Wang et al. ${ }^{19)}$ Wagner and Pierre ${ }^{20)}$ calculated $\Delta H^{m}$ from the ion intensity ratios measured with a Knudsen cell-mass spectrometer combination. They found $\Delta H^{m}$ to be symmetric and regular.

Using the associate model presented we calculated the following parameters for the temperature dependent description of $\Delta H^{m}$ in the system Ti-Fe:

$$
\begin{aligned}
& C_{1}=-58.84 \mathrm{~kJ} \cdot \mathrm{mol}^{-1}, \quad C_{2}=-7.82 \mathrm{~kJ} \cdot \mathrm{mol}^{-1}, \\
& C_{3}=-26.72 \mathrm{~kJ} \cdot \mathrm{mol}^{-1}, \quad \Delta H_{\mathrm{TiFe}}^{\mathrm{F}}=-72.23 \mathrm{~kJ} \cdot \mathrm{mol}^{-1} \\
& \text { and } \Delta S_{\mathrm{TiFe}}^{\mathrm{F}}=-22.37 \mathrm{~J} \cdot \mathrm{mol}^{-1} \cdot \mathrm{K}^{-1} .
\end{aligned}
$$

The standard deviation between the experimental data and the adapted curve amounts to $\sigma=0.267$.

With Eq. (11) the excess heat capacity was calculated. The variation of $\Delta C_{p}^{A B}(x s)$ with the composition is given for 1950 and $2112 \mathrm{~K}$ in Fig. 4. The maxima of the curves represent the concentration of the strongest atomic interactions in the melt. With help of the GibbsHelmholtz function (20) the free energy of mixing $\Delta G^{m}$ was calculated:

$$
\Delta G^{m}=\Delta H^{m}-T \Delta S^{m}
$$

Figure 5 shows the resulting curve for $\Delta G^{m}$ at $2031 \mathrm{~K}$. Furthermore, the partial free energies of mixing $\Delta \bar{G}_{i}$ of

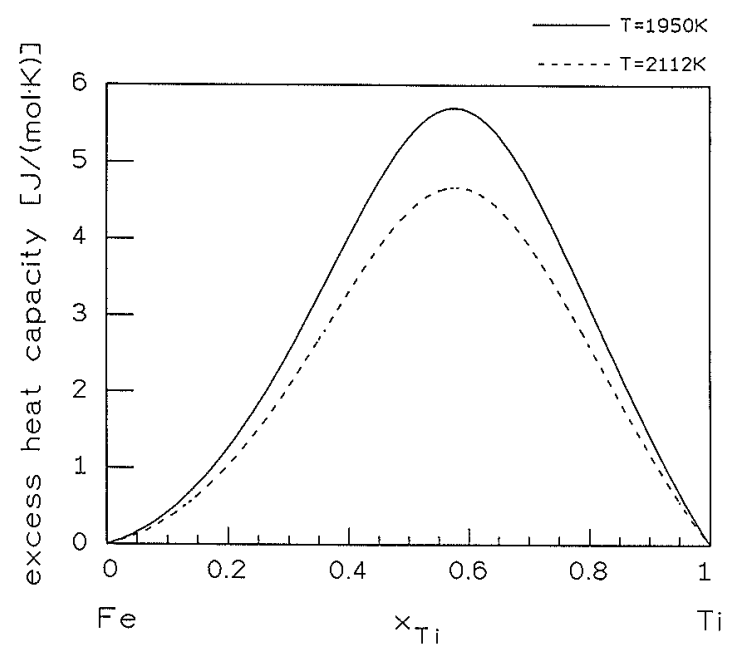

Fig. 4. Excess heat capacities $\Delta C_{n}^{A B}(x s)$ of liquid iron-titanium alloys calculated with the associate model. iron and titanium can be derived according to Eq. (21).

$$
\Delta \bar{G}_{i}=\Delta G+\left(1-x_{i}\right) \frac{d \Delta G}{d x_{i}}
$$

Both results are also given in Fig. 5 and Table 1.

The course of the free energy of mixing is more symmetric than that of the mixing enthalpy. This is caused by the entropy (Eq. (17)).

According to Eq. (22) the partial free energies of mixing were converted into activities.

$$
\Delta \bar{G}_{i}=R T \ln a_{i}
$$

Figure 6 and Table 1 show the result of the calculation for $T=1873 \mathrm{~K}$. The curves are in excellent agreement with the experimental data given by Furukawa and $\mathrm{Kato}^{21)}$ who determined the activities in the system $\mathrm{Fe}-\mathrm{Ti}$ directly by means of the Knudsen cell-mass spectrometer combination at $T=1873 \mathrm{~K}$. Not least, this correspondence confirms our mixing enthalpy measurements. Furukawa and Kato calculated mixing enthalpies from their Knudsen cell measurements. These values are also presented in Fig. 3. Together with the measurements of Wagner and Pierre ${ }^{20)}$ one can follow that the Knudsen

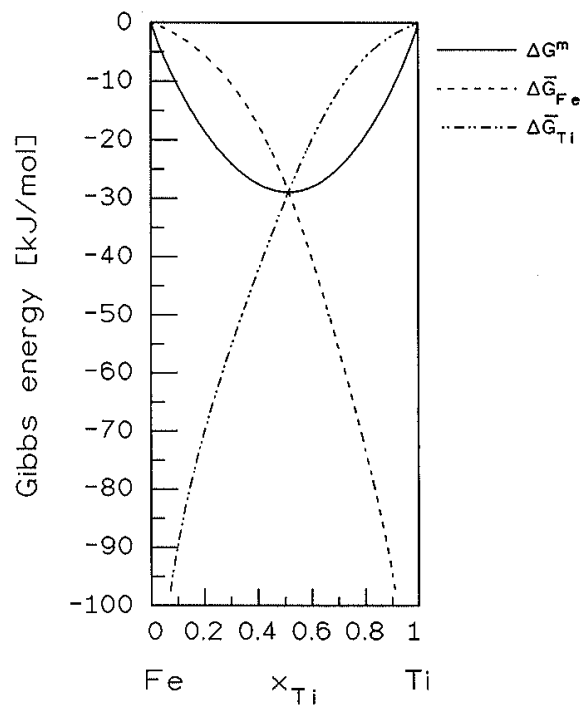

\begin{tabular}{|c|c|c|c|c|c|c|}
\hline$x_{\mathrm{Ti}}$ & $\begin{array}{c}\Delta H^{m} \\
{\left[\mathrm{~kJ} \cdot \mathrm{mol}^{-1}\right]}\end{array}$ & $\begin{array}{c}\Delta G^{m} \\
{\left[\mathrm{~kJ} \cdot \mathrm{mol}^{-1}\right]}\end{array}$ & $\begin{array}{c}\Delta \bar{G}_{\mathrm{Ti}} \\
{\left[\mathrm{kJ} \cdot \mathrm{mol}^{-1}\right]}\end{array}$ & $\begin{array}{c}\Delta \bar{G}_{\mathrm{Fe}} \\
{\left[\mathrm{kJ} \cdot \mathrm{mol}^{-1}\right]}\end{array}$ & $\gamma_{\mathrm{Fe}}$ & $\gamma_{\mathrm{Ti}}$ \\
\hline 0 & 0 & 0 & $-\infty$ & 0 & 1 & 0.018 \\
\hline 0.1 & -6.02 & -10.84 & -89.41 & -2.11 & 0.97 & 0.03 \\
\hline 0.2 & -11.44 & -18.39 & -70.20 & -5.44 & 0.88 & 0.05 \\
\hline 0.3 & -16.15 & -23.89 & -55.58 & -10.31 & 0.74 & 0.09 \\
\hline 0.4 & -19.83 & -27.43 & -42.64 & -17.29 & 0.55 & 0.16 \\
\hline 0.5 & -21.90 & -28.89 & -30.70 & -27.09 & 0.35 & 0.28 \\
\hline 0.6 & -21.67 & -28.09 & -20.12 & -40.03 & 0.19 & 0.46 \\
\hline 0.7 & -18.86 & -24.90 & -11.77 & -55.54 & 0.09 & 0.67 \\
\hline 0.8 & -13.87 & -19.36 & -5.93 & -73.08 & 0.05 & 0.85 \\
\hline 0.9 & -7.42 & -11.42 & -2.18 & -94.55 & $2.3 \cdot 10^{-3}$ & 0.96 \\
\hline 1.0 & 0 & 0 & 0 & $-\infty$ & 0.0121 & 1 \\
\hline
\end{tabular}

Fig. 5. Calculated integral free energy of mixing $\Delta G^{m}$ and partial free energies of mixing $\Delta \bar{G}_{i}$ of iron and titanium in liquid iron-titanium alloys $(T=1873 \mathrm{~K})$, liquid reference.

Table 1. Thermodynamic properties of mixing in the liquid system iron-titanium ( $T=1873 \mathrm{~K}$ ), liquid reference. 


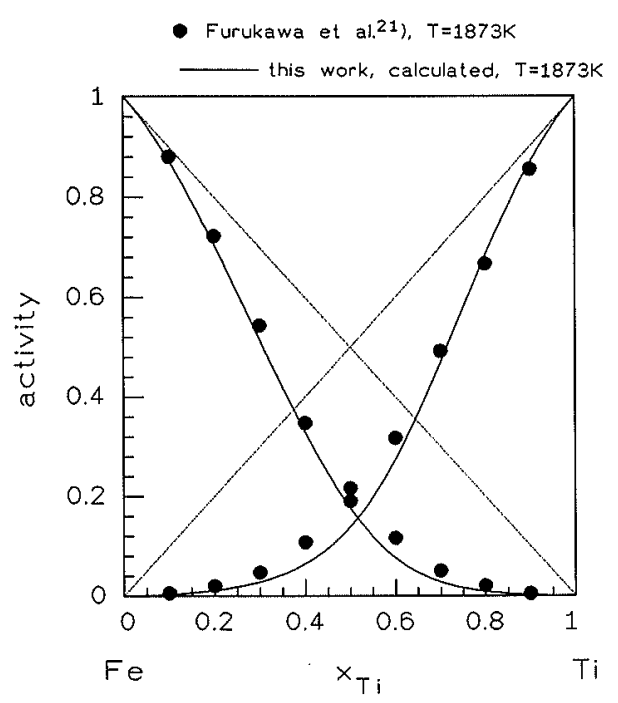

Fig. 6. Calculated activities of iron and titanium in liquid iron-titanium alloys $(T=1873 \mathrm{~K})$ together with experimental values, ${ }^{21)}$ liquid reference.

method for evaluating $\Delta H^{m}$ has to be regarded critically due to the fact that slightly differing temperature increments of the ion current ratios lead to drastical changes in the mixing enthalpies.

\section{Conclusions}

Measurements by levitation alloying calorimetry were performed to determine the mixing enthalpies in the liquid state of the system iron-titanium. The regular associate model was adapted to describe the exothermic temperature dependent mixing enthalpies of this compound forming system. The activities of iron and titanium which were calculated on the basis of this model are in agreement with the experimental data given by Furukawa and Kato. ${ }^{21)}$ This demonstrates the strength of pur calorimetric measurements combined with suitable thermodynamic evaluation.

\section{Acknowledgement}

We wish to thank the Deutsche Forschungsgemeinschaft (DFG) for their financial support.

\section{REFERENCES}

1) R. H. Wiswall and J. J. Reilly: Inorg. Chem., 11 (1972), 1691.

2) I. W. Donald and H. A. Davies: J. Non-Cryst. Solids, 30 (1978), 77.

3) O. Kubaschewski: Iron-Binary Phase Diagrams, SpringerVerlag, Berlin, Heidelberg, New York, Verlag Stahleisen m.b.H. Düsseldorf, (1982).

4) B. Predel: Heterogene Gleichgewichte-Grundlagen und Anwendungen, Steinkopff Verlag Darmstadt, (1982).

5) F. Sommer: Z. Metallkd., 73 (1982), 77.

6) M. G. Frohberg and G. Betz: Arch. Eisenhüttenwes., 51 (1980), 235.

7) E. Arpaci and M. G. Frohberg: Z. Metallkd., 76 (1985), 440.

8) R. Lin and M. G. Frohberg: High Temp.-High Press., 24 (1992).

9) K. Schaefers, J. Qin and M. G. Frohberg: Steel Res., 64 (1993), 229.

10) K. Schaefers, M. Rösner-Kuhn, J. Qin and M. G. Frohberg: Steel Res., 66 (1995), 183.

I1) K. Schaefers, J. Qin, M. Rösner-Kuhn and M. G. Frohberg: Can. Metall. Q., in press.

12) M. Rösner-Kuhn, J. Qin, K. Schaefers, U. Thiedemann and M. G. Frohberg: Z. Metallkd, 86 (1995), in press.

13) K. Schaefers: Ph.D. Thesis, Technische Universität Berlin, Germany, (1993).

14) J. Qin, M. Rösner-Kuhn, K. Schaefers and M. G. Frohberg: $Z$. Metallkd., 85 (1994), 692.

15) A. S. Jordan: Calculation of Phase Diagram and Thermochemistry of Alloy Phases, ed. by Y. A. Chang and J. F. Smith, Metallurgical Society of AIME, Warrendale, PA, (1979), 100.

16) J. Qin, R. Lin, K. Schaefers and M. G. Frohberg: Z. Metallkd., 84 (1993), 675.

17) Yu. O. Esin, M. G. Valishev, A. F. Ermakov, P. V. Gel'd and M. S. Petrushevskiy: Russ. Metall, 3 (1981), 15.

18) G. I. Batalin, V. P. Kurach and V. S. Sudavtsova: Russ. J. Phys. Chem., 58 (1984), 289.

19) H. Wang, R. Lück and B. Predel: Z. Metallkd., 82 (1991), 659.

20) S. Wagner and G. R. St. Pierre: Metall. Trans., 3 (1974), 887.

21) T. Furukawa and E. Kato: ISIJ Int., 16 (1976), 382. 